\title{
Development of an auditory implant manipulator for minimally invasive surgical insertion of implantable hearing devices
}

\author{
C STIEGER $^{1,2}$, M CAVERSACCIO $^{2}$, A ARNOLD $^{2}$, G ZHENG $^{1}$, J SALZMANN $^{1}$, D WIDMER ${ }^{1}$,

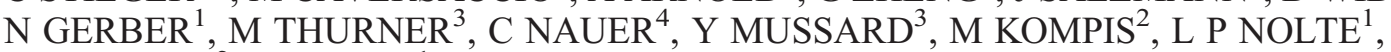

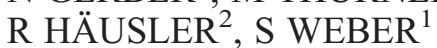 \\ ${ }^{1}$ ARTORG Center for Biomedical Engineering Research, University of Bern, ${ }^{2}$ University Department of ENT, Head \\ and Neck Surgery, Inselspital Bern, ${ }^{3}$ Microrobotics Laboratory, University of Applied Science, Biel, and ${ }^{4}$ Institute \\ for Diagnostic and Interventional Neuroradiology, Inselspital, University of Bern, Switzerland
}

\begin{abstract}
Objective: To present the auditory implant manipulator, a navigation-controlled mechanical and electronic system which enables minimally invasive ('keyhole') transmastoid access to the tympanic cavity.

Materials and methods: The auditory implant manipulator is a miniaturised robotic system with five axes of movement and an integrated drill. It can be mounted on the operating table. We evaluated the surgical work field provided by the system, and the work sequence involved, using an anatomical whole head specimen.

Results: The work field provided by the auditory implant manipulator is considerably greater than required for conventional mastoidectomy. The work sequence for a keyhole procedure included pre-operative planning, arrangement of equipment, the procedure itself and post-operative analysis.

Conclusion: Although system improvements are necessary, our preliminary results indicate that the auditory implant manipulator has the potential to perform keyhole insertion of implantable hearing devices.
\end{abstract}

Key words: Robotics; Minimally Invasive Surgical Procedures; Implantable Hearing Systems; Cochlear Implants; Temporal Bone

\section{Introduction}

Surgical insertion of cochlear implants or other implantable hearing systems (e.g. the Direct Acoustical Cochlear Stimulation system) requires access to the tympanic cavity. Currently, a mastoidectomy procedure is considered to represent the best possible access for such implantations, as it enables the identification of landmarks and structures at risk (e.g. the facial nerve, chorda tympani, lateral semicircular channel and posterior wall of the external auditory canal). Alternative procedures have been developed, such as the suprameatal approach ${ }^{1}$ and the pericanalar approach, ${ }^{2}$ in order to reduce the extent of the approach and to minimise the risk of a facial nerve lesion.

Several studies have indicated that the use of imageguided systems and robot-assisted surgery may help increase intra-operative patient safety during some ENT procedures. ${ }^{3-5}$ The American Academy of Otolaryngology has recommended the use of such systems in selected surgical cases, such as skull base surgery and revision surgery of the paranasal sinus. ${ }^{6}$ Recent studies have experimentally assessed the use of image-guided systems and robot-assisted techniques in otological surgery. Specific designs have been proposed for minimally invasive cochleostomy ${ }^{7,8}$ and micro-fenestration of the stapedial footplate. ${ }^{9}$ Several approaches for minimally invasive mastoidectomy and keyhole procedures have been studied, using either image-guided systems only, ${ }^{10}$ image-guided systems plus individual adapted drilling guides, ${ }^{11}$ or mechanical and electronic systems based on industrial robots. ${ }^{12-14}$ Industrial robots have the drawback of not having been developed according to the surgeon's needs, nor with the intention of integration into the surgical work sequence. Dedicated development within a surgical context could enable the integration of basic surgical requirements, such as simplicity of use in the operating theatre, reduced risk of complication, shorter operative time and lower cost.

We aimed to develop a miniaturised robotic system to be used during the surgical insertion of implantable hearing devices, with a focus on surgical integration. To this end, we describe below the technical and surgical concepts involved, a work field evaluation, 
integration of the system into the surgical work sequence, and an initial ex vivo experiment to investigate the overall accuracy of the system.

This system was named the auditory implant manipulator.

\section{Materials and methods \\ Robot}

The miniaturised robot enabled movement with five degrees of freedom, being a combination of a socalled bipod structure, a double scara structure (Figure 1) and a tool axis (the $z$ axis).

The first two axes of movement were arranged as a bipod subsystem (Figure 1). Two parallel arms could be moved individually, allowing the double scara subsystem to operate in rotational and pitched movements around axes $x$ and $y$, respectively. Thus, the robot was able to move along the surface of a sphere that had dimensions matching those of a human head. The designed range of motion was $\pm 16^{\circ}$ roll and $35-53^{\circ}$ pitch. The double scara subsystem provided translational motion of the tool axis. It comprised two active axes providing effective movement in a plane of $60 \times$ $60 \mathrm{~mm}$ (see $\mathrm{x}^{\prime}$ and $\mathrm{y}^{\prime}$ in Figure 1). The tool axis provided an effective motion of $150 \mathrm{~mm}$ along the working axis $z^{\prime \prime}$. An integrated snap closure enabled easy mounting or removal of a drill used during otological microsurgery (Bienair, Noirement, Switzerland), to facilitate cleaning and sterilisation. Burrs could be changed while the drill was in the mounted position.

All five axes were equipped with digital differential encoders (with a resolution of 512 ticks per revolution on three channels; TTL Maxon, Sachseln, Switzerland) and were controlled with a stand-alone bare bone system (MSX-Box, ADDI-DATA, Rheinmuenster, Germany) running on a Linux kernel with a real-time extension. The control structure provided positional

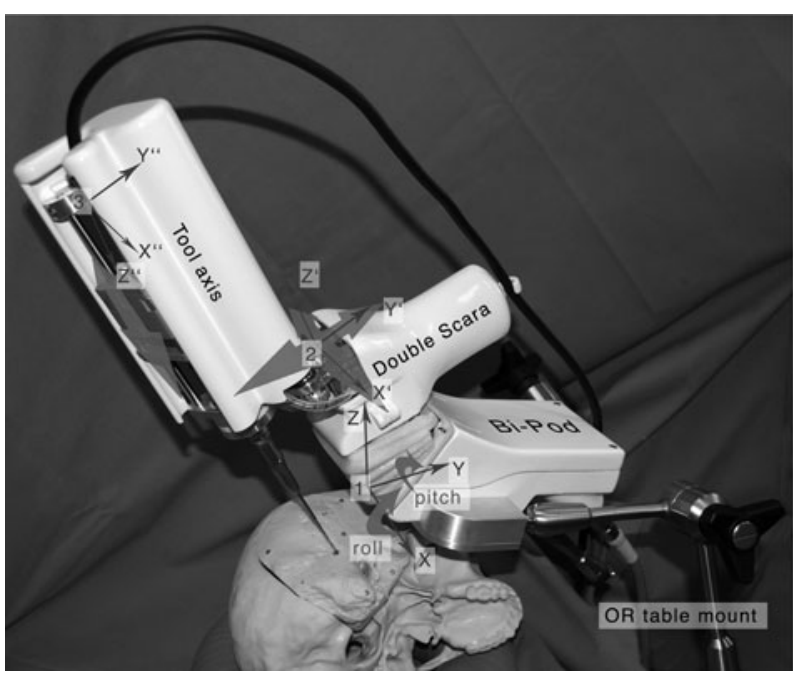

FIG. 1

Prototype of the auditory implant manipulator. $\mathrm{OR}=$ operating room proportional-integral-derivative control $(\mathrm{f}=800 \mathrm{~Hz}$ ) for every axis. The axis controllers provided a speed proportional voltage signal to the connected drive amplifiers $(\mathrm{f}=4 \mathrm{kHz})$ (Elmo Violin, Petchtikva, Israel). The auditory implant manipulator could be controlled by computer or manually using a three-dimensional mouse (SpaceNavigator; 3D Connexion, Fremont, California, USA).

The miniature robot was designed with a focus on its future surgical application and integration. Therefore, a number of clinically critical issues were considered. The base of the robot system was mounted onto the operating table using two standard clinical articulated arms (Fisso; Baitella, Zürich, Switzerland), as shown in Figure 1, allowing arbitrary placement and orientation. The complete structure could then be positioned manually in the work area. For improved rigidity, the two articulated arms were placed as far apart from each other as possible on the operating table. Figure 1 shows one of the arms in the foreground, while the other one is placed behind the bipod structure. The housing was designed to be easily coverable with sterile drapes.

\section{Work field evaluation}

Miniaturising a robotic system reduces the work space it requires. However, we needed to ensure that the auditory implant manipulator provided a sufficient surgical work field to accommodate all the steps of the implantation procedure.

The work field of the auditory implant manipulator resembled a $90^{\circ}$ section of a cylinder with a radius of $55 \mathrm{~mm}$ and a height of $65 \mathrm{~mm}$ (Figure 2a). This work field was compared with the dimensions of the housings of five cochlear implants and implantable hearing systems: the Nucleus Freedom (Cochlear, Sydney, Australia), Vibrant Soundbridge (Medel, Innsbruck, Austria), Pulsar (Vibrant Medel, Innsbruck, Austria), Carina (Otologics, Boulder, Colorado, USA) and HiRes (Advanced Bionics, Valencia, CA, USA). The work field was also compared with the dimensions of an exhaustive mastoidectomy, using a life-sized plastic temporal bone (Pettigrew, Glasgow, Scotland, UK) (Figure 2b).

\section{Accuracy of auditory implant manipulator}

The accuracy of the auditory implant manipulator was evaluated using a plastic model with a carved surface similar to the mastoid portion of the temporal bone (Figure 3a).

The base plate of the model was aligned with the $x-y$ plane of the auditory implant manipulator. A $5 \times 5$ points grid was positioned on the central part of the model. At each grid position, a trajectory of a defined angle and depth was planned (Figure 3b). The pitch and roll angles were decremented by $3^{\circ}$ along the $x$ and $y$ axes, respectively, for each trajectory (Figures 1 and $4 b$ ). 
(a)

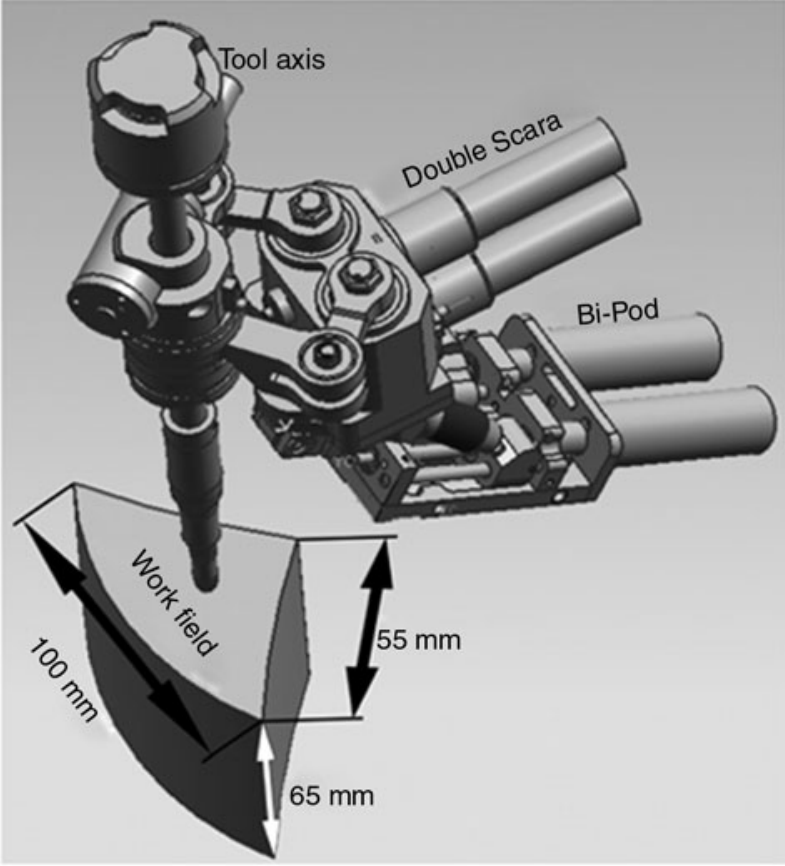

(b)

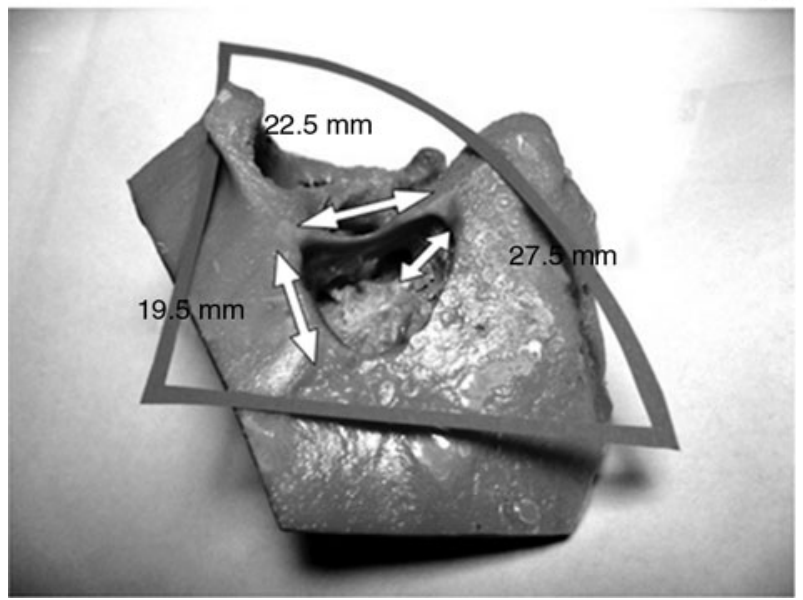

FIG. 2

(a) Work field provided by the auditory implant manipulator, and (b) relation of this work field to a mastoidectomy site, as indicated using a life-sized plastic temporal bone.

To evaluate the accuracy of robot-assisted drilling, an active optical tracking system was used (OptoTrak 3020; Northern Digital, Waterloo, Ontario, Canada). A custom-made trajectory digitisation probe was designed to determine the position of the end-point of a drilled trajectory as well as its axial orientation. A dynamic reference base ${ }^{15}$ was attached to the test model in order to define a local co-ordinate system. Figure 4 shows the experimental arrangement for trajectory digitisation. Using the digitised position of the end point and the orientation of each drilled hole together with its planned depth, the position of the entry point of the associated hole was calculated. We could then calculate the angular difference between any two neighbouring holes in the $x$ or $y$ direction, as well as the distance between their entry points. These

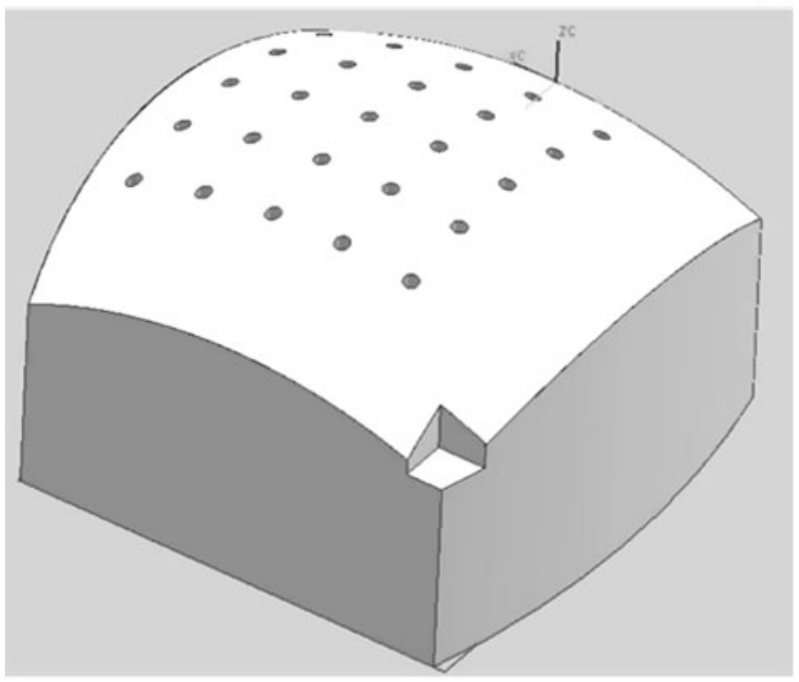

(b)

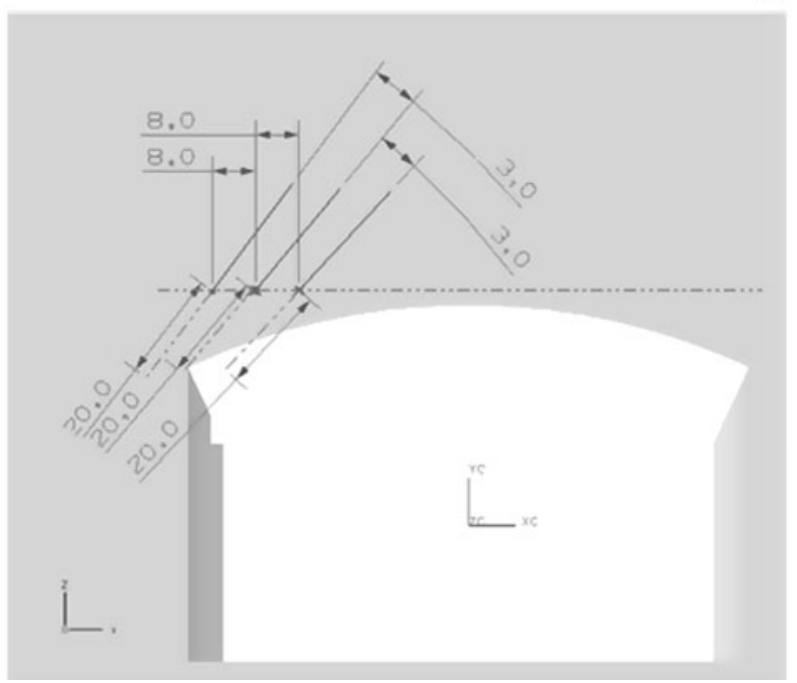

FIG. 3

(a) Plastic model for accuracy measurement; (b) side view of planned drilling trajectories.

values were compared with the planned values, and the rotational and translational accuracy of the robot was determined.

\section{Work sequence}

The clinical facility of the auditory implant manipulator to perform a 'keyhole' procedure was evaluated using an anatomical whole head specimen. The use of human anatomical specimens was approved by the Bernese cantonal ethical commission (approval number KEK-BE 030/08).

We undertook pre-operative computed tomography (CT) scanning, pre-operative planning, execution of the keyhole procedure, post-operative CT scanning and analysis of accuracy, as described in detail below.

Pre-operatively, two axial CT scans were taken with an eight-slice multidetector CT scanner (Light Speed Ultra; GE Medical Systems, Milwaukee, Wisconsin, USA). First, a CT scan of the entire skull was 


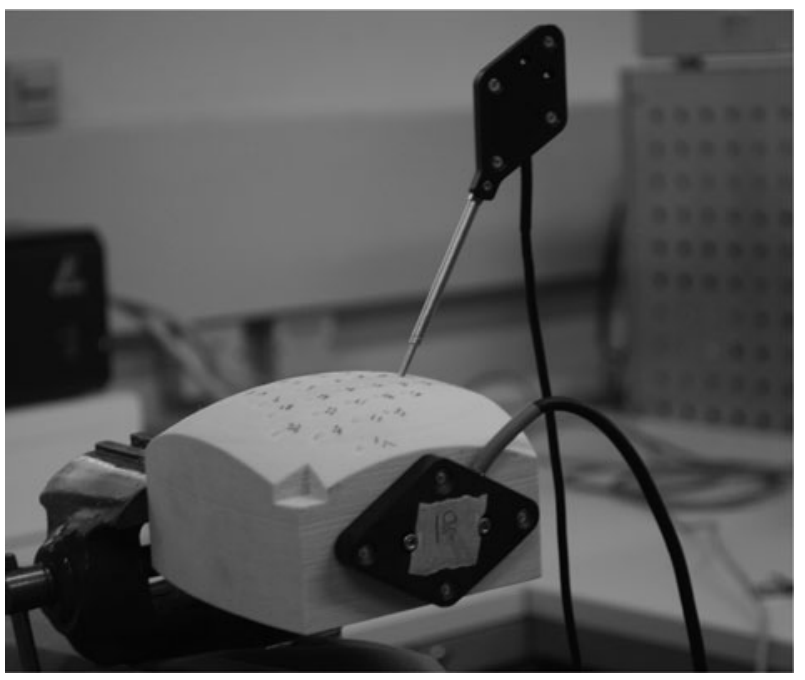

FIG. 4

Experimental arrangement for accuracy measurement, using active infrared based navigation with a custom-made probe.

performed with a slice thickness of $1.25 \mathrm{~mm}$. Image data were reconstructed with 50 per cent overlap, resulting in an effective axial spatial resolution of $0.625 \mathrm{~mm}$. These data were loaded into the navigation system for later registration of the head and visualisation of the drilling tool. The second scan included only the temporal bone. Images of $0.625 \mathrm{~mm}$ thickness were acquired. Reconstruction of the slices with an overlap of 68 per cent resulted in a very high axial resolution of $0.2 \mathrm{~mm}$, rendering in sufficient detail tiny anatomical features of the temporal bone, such as the facial nerve.

Based on these data, pre-operative planning of the keyhole procedure was undertaken by a neuroradiologist, using planning software of our own design (Figure 5). This software had two basic functions: segmentation of important structures and trajectory planning. Four different segmentation modes were used for this work sequence experiment. First, a threshold was set to segment high density bony structures of the temporal bone. Then, a region growing algorithm was used to automatically segment the incus and malleus. The stapes was reconstructed in the shape of a cone by defining the stapes head and two points at the oval window. Finally, the facial nerve was segmented by selecting a set of single points that were interpolated by the software (Figure 5a) and visually inspected by the neuroradiologist.

The trajectory of the keyhole procedure was defined by an entry point and a target point. The target point was set directly at the round window (Figure 5a). The entry point was then selected on the bony surface behind the ear, in such a way that the resulting trajectory circumvented the facial nerve and remained behind the external auditory canal (Figure 5b).

Data from the above-described planning and CT scans were loaded into navigation software of our own design (named Marvin). ${ }^{16}$ The anatomical whole head specimen was mounted on an operating table using a Mayfield clamp (Scherrer Mayfield, Cincinnati, Ohio, USA). The auditory implant manipulator was mounted onto the operating table and initial alignment was performed. Two reference marker shields, for the drill and the specimen, were tracked using an infrared camera system (Polaris; Northern Digital, Kalamazoo, MI, USA) (Figure 6a). The robot marker shield was attached to the tool axis and calibrated. The second reference marker shield was mounted onto the anatomical whole head specimen using a dental bite-block, providing non-invasive fixation.

Surface matching based registration included samples of the surfaces of the frontal, parietal and temporal bones (Figure 6b). The entry point was located with a navigated pointer and the bone was exposed locally (Figure 6a).

The axis of the navigated drill was aligned to the axis of the planned trajectory, and the tip of the drill was positioned at the entry point of the planned trajectory using the three-dimensional mouse. The keyhole access site was then drilled automatically with a $1.6 \mathrm{~mm}$ twist drill (Leibinger; Stryker, Valencia, California, USA), at $20000 \mathrm{rpm}$ and with a constant feed rate of $0.1 \mathrm{~mm} / \mathrm{sec}$ (Figure $6 \mathrm{c}$ ).

Following drilling of the keyhole site, a post-operative CT scan was taken to compare the planned and the actual keyhole drill sites. Amira software (Visage Imaging, San Diego, California, USA) was used to match the pre- and post-operative CT scans and to determine the co-ordinates of the planned and actual drill trajectories (Figure 7). Differences between the two trajectories were calculated using Matlab version R2007a software (Mathworks; Natick, Massachusetts, USA). Finally, a mastoidectomy was performed manually for visual inspection.

\section{Results}

\section{Work field}

The bone bed area required for implantation of all the examined implants was smaller than the upper surface made available within the work field of the auditory implant manipulator (Figure 2a). Even the capsule of the Carina fully implantable hearing system (Otologics), which required the largest bone bed $(42 \times 27 \mathrm{~mm})$, fitted into the work field (Figure $2 \mathrm{~b}$ ).

Even the largest possible mastoidectomy dimensions, as applied to a life-sized plastic temporal bone model (i.e. supero-inferior $22.5 \mathrm{~mm}$, antero-posterior $19.5 \mathrm{~mm}$ and proximo-distal $27.5 \mathrm{~mm}$ ) were significantly smaller than the auditory implant manipulator work field, for any rotational configuration.

The auditory implant manipulator work field was larger than the size required for either mastoidectomy or implant device bone bed exposure; however, in order to perform both surgical procedures in sequence, the robot would require repositioning. 
(a)

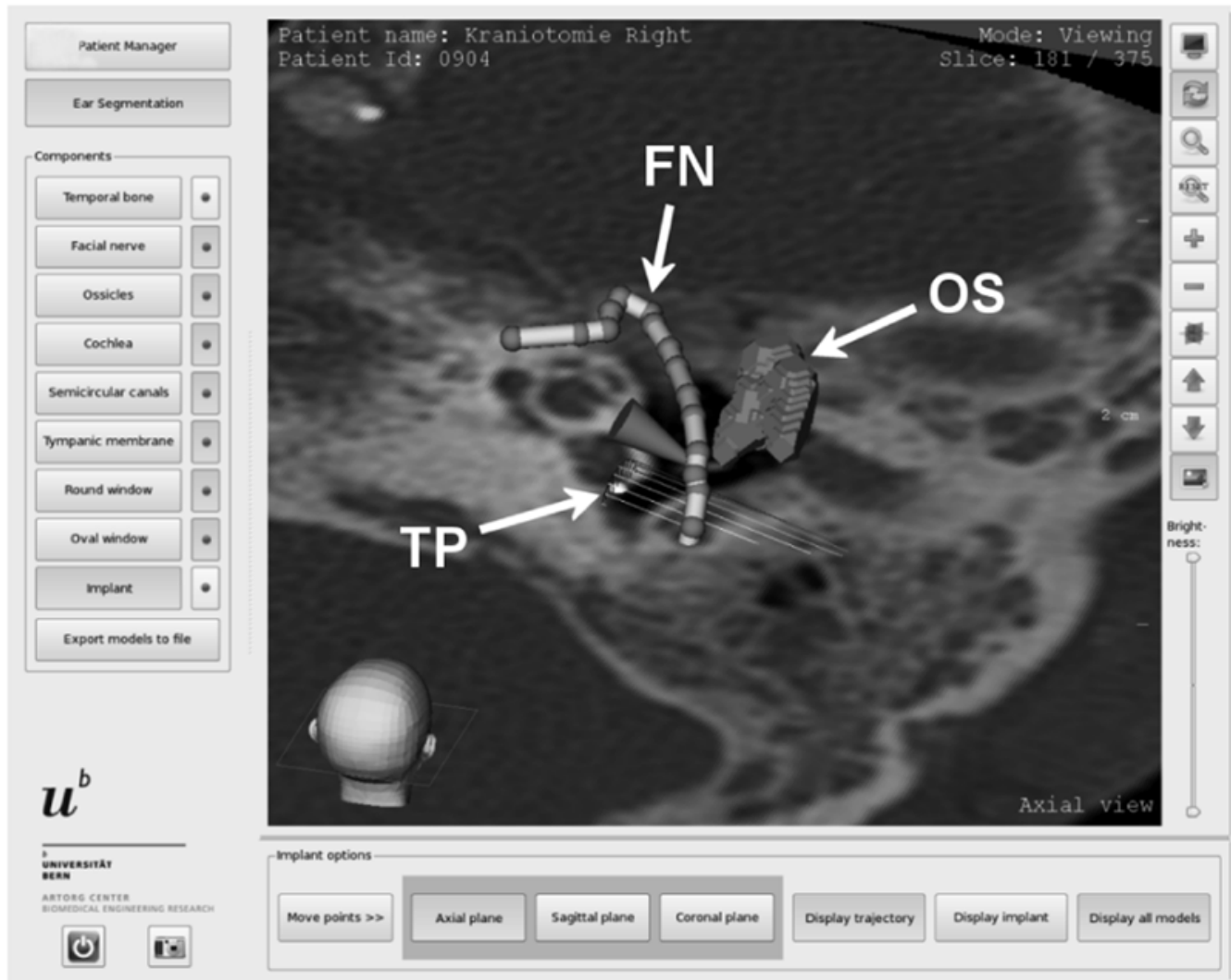

(b)

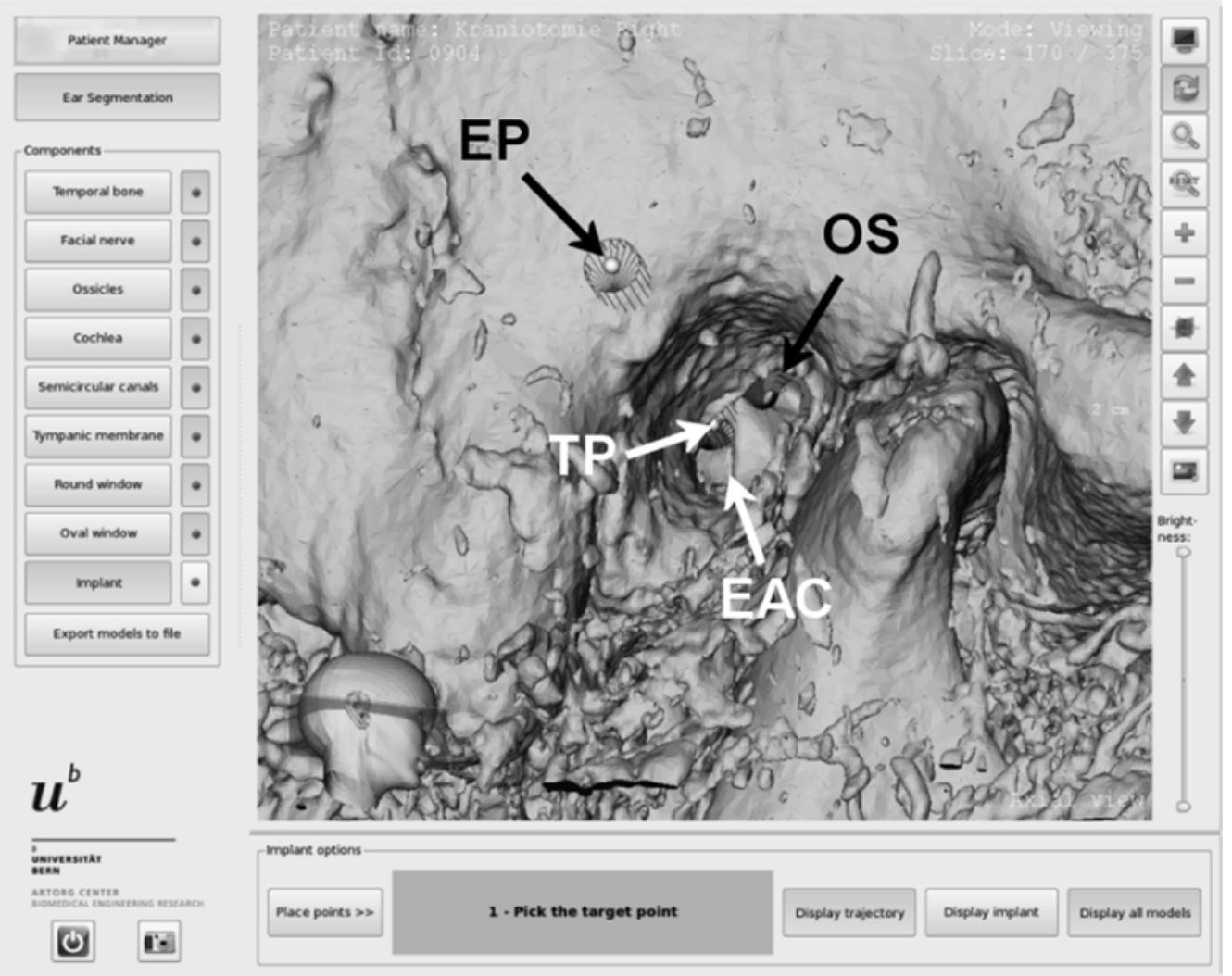

FIG. 5

(a) Screengrab of planning software, with segmented ossicles (OS), facial nerve (FN) and target point (TP) on the round window. (b) Screengrab of planning software, showing a view through the external auditory canal (EAC), and the entry point (EP). 
(a)

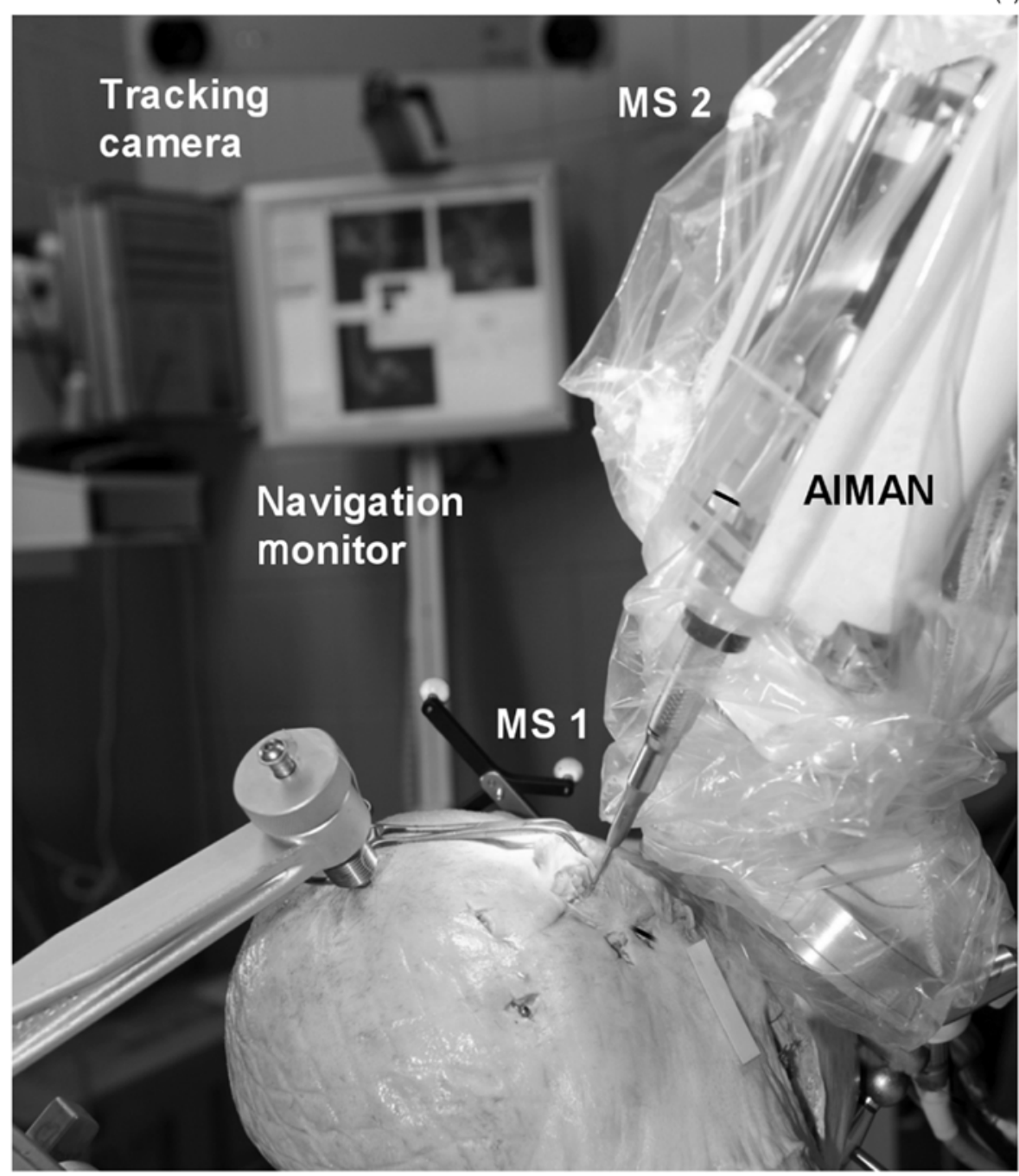

(b)

(c)
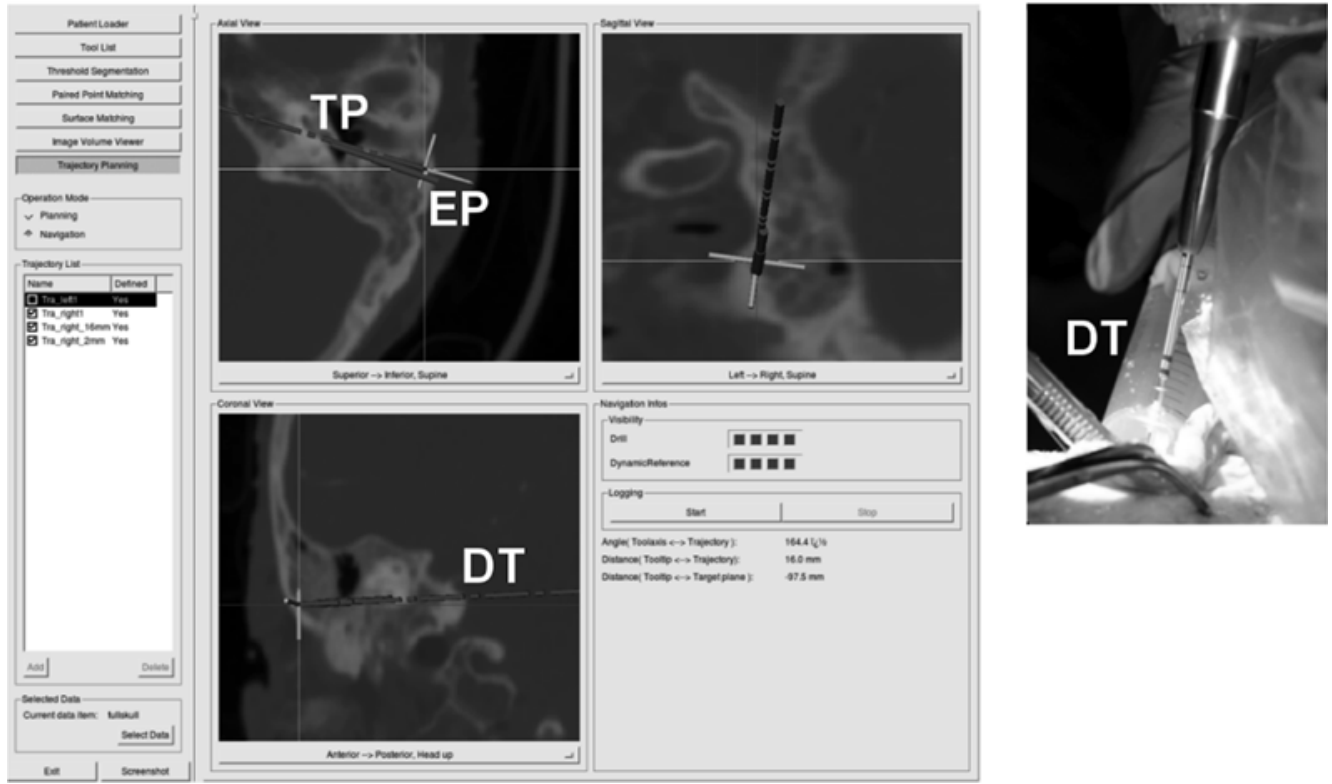

FIG. 6

(a) Experimental arrangement for the 'keyhole' drilling procedure. The relative positions of the marker shields of the auditory implant manipulator (AIMAN; MS 2) and the anatomical whole head (MS 1) are tracked by an infrared camera. (b) Screengrab of navigation monitor (seen in the background of Figure 6a), showing views of the drilling tool (DT) and the planned trajectory between entry point (EP) and target point (TP). (c) Closer view of the drilling tool. 


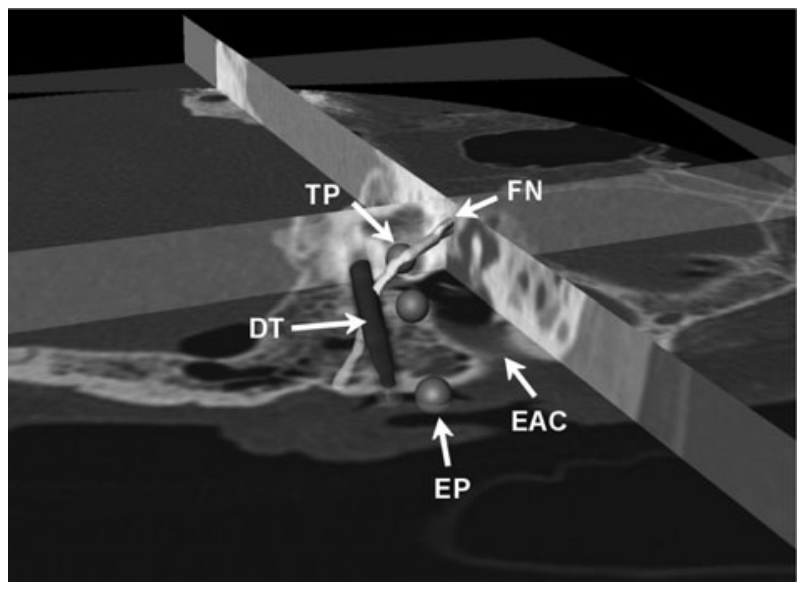

FIG. 7

Comparison of the pre-operatively planned entry and target points, versus the post-operatively assessed drill trajectory. $\mathrm{TP}=$ target point; $\mathrm{FN}=$ facial nerve; $\mathrm{DT}=$ drilled trajectory; $\mathrm{EAC}=$ external auditory canal; EP = entry point

\section{Robot accuracy}

The rotational and translational errors encountered while the robot was moved along the $x$ and $y$ axes are summarised in Table I. Presenting results as mean \pm standard deviation, a rotational error of $0.4 \pm 0.3^{\circ}$ and a translational error of $1.2 \pm 0.5 \mathrm{~mm}$ were found along the $x$ axis. Along the $y$ axis, the rotational error was changed to $0.6 \pm 0.7^{\circ}$ and the translational error to $1.6 \pm 0.4 \mathrm{~mm}$. The overall rotational accuracy was $0.6 \pm 0.6^{\circ}$ and the overall translational accuracy $1.4 \pm 0.5 \mathrm{~mm}$.

\section{Work sequence}

The work sequence for the keyhole procedure was performed on an anatomical whole head specimen, and included pre-operative planning (Figure 5), arrangement of experimental equipment (Figure 6) and postoperative analysis (Figure 7).

Segmentation of the bony structure, facial nerve and ossicles took less than 30 minutes. Planning of a straight trajectory of $1.6 \mathrm{~mm}$ thickness was possible without contacting the facial nerve or the external auditory canal (Figure 5a and 5b).

The robot could have been mounted by an assistant from the rear side of the operational table in such a

\begin{tabular}{llccc}
\multicolumn{5}{c}{ TABLE I } \\
\multicolumn{5}{c}{ ACCURACY STUDY: TRANSLATIONAL AND } \\
ROTATIONAL ERRORS \\
Error & Axis & Mean \pm SD & Min & Max \\
\hline \multirow{2}{*}{ Translnl (mm) } & $x$ & $1.2 \pm 0.5$ & 0.4 & 2.7 \\
& $y$ & $1.6 \pm 0.4$ & 0.8 & 2.4 \\
Rotnl $\left({ }^{\circ}\right)$ & $x$ & $0.4 \pm 0.3$ & 0.1 & 0.9 \\
& $y$ & $0.6 \pm 0.7$ & 0.0 & 3.2 \\
\hline
\end{tabular}

$\mathrm{SD}=$ standard deviation; $\min =$ minimum $; \max =$ maximum; translnl = translational; rotnl $=$ rotational way that the surgeon had direct, free access to the operative field at all times.

The total registration error of surface matching was $0.2 \mathrm{~mm}$. After alignment of the auditory implant manipulator to the planned trajectory, drilling of the keyhole access site took less than 5 minutes (Figures 6).

Figure 7 shows the superimposed pre- and post-operative CT scans, together with the planned trajectory (entry point and target point) and the drilled keyhole site. When the planned and drilled trajectories were compared, there was a difference of $3.42 \mathrm{~mm}$ minimal distance and $8.4^{\circ}$ angle. The actual drilled keyhole site was situated well behind the external auditory canal but touched the facial nerve. This result was confirmed by opening the middle-ear cavity during manual mastoidectomy.

\section{Discussion}

We present the auditory implant manipulator, a compact and flexible miniaturised robotic system for use during the surgical insertion of cochlear implants and other implantable hearing devices. This small, light system is designed to be mounted on the rear side of the operating table, giving the surgeon maximum free access to the operative field. As a direct consequence of its small size, the robot provides only a limited surgical work field. Therefore, the present study was conducted to compare the work field of the auditory implant manipulator with the dimensions of a conventional mastoidectomy and the size of the bone bed required for cochlear implantation. The work field was found to be sufficient for both surgical procedures, with repositioning. These two implantation steps are not necessarily executed one after the other; thus, it is expected that the surgeon would prefer to remove the robot from the surgical field after the first step. The implant bed may then be drilled without navigation. Thus, pre-operative navigational system registration is only required for mastoidectomy drilling. Further realignment of the auditory implant manipulator can be performed quickly by a surgical nurse in non-sterile attire, due to the device's articulated arms, and therefore should not delay the surgical procedure.

The auditory implant manipulator's translation accuracy (i.e. $1.4 \pm 0.5 \mathrm{~mm}$ ) is equivalent to other robotic systems designed for orthopaedic surgery, such as the PathFinder (Armstrong Healthcare, High Wycombe, UK) and the NeuroMate (Integrated Surgical Systems (ISS), Sacramento, CA, USA). However, the application of such technology to transmastoidal temporal bone surgery requires an accuracy of less than $1 \mathrm{~mm}$. Part of our system's error may have arisen from the chosen measurement method: the digitalisation probe could not be held tightly in all drilled holes because of limited depth. A more accurate measurement tool, such as a co-ordinate measuring machine, may have improved determination of the accuracy of the auditory implant manipulator. 
The clinical work sequence undertaken when performing a keyhole procedure using the auditory implant manipulator was evaluated using an anatomical whole head specimen. This experiment demonstrated successful integration of the auditory implant manipulator with an image-guided navigation system. The presented work sequence included two pre-operative axial CT scans. Such scanning has two advantages: (1) large portions of the skull surface are available for registration, and (2) high resolution scanning can be used for segmentation of important temporal bone structures. It could be argued that taking two CT scans is inappropriate for cochlear implant surgery patients because of the associated increased radiation exposure. However, it has been shown that low-dose CT scans can be used for computer-assisted navigation; the radiation dose can therefore be substantially reduced, compared with the dose received during diagnostic CT scanning. ${ }^{17}$

The planning software used enabled interactive segmentation of most of the relevant important structures, such as the facial nerve, the round window and the posterior wall of the external auditory canal. The trajectory was then defined by the target point and the entry point. These two points must be carefully chosen so that the trajectory passes inside the angle formed by the bony part of the chorda tympani and the facial nerve (mean value $\left.21.7^{\circ}\right),{ }^{18}$ and posterior to the external auditory canal. Alternatively, an atlas-based automated trajectory planning system based on statistical models could be used, as described by Labadie et al. ${ }^{11,19}$

- Several possibilities have been proposed for minimally invasive mastoidectomy surgery: image-guided surgery; image-guided surgery plus drilling guides; and mechanical and electronic systems based on industrial robots

- This paper presents a miniaturised robotic system, the auditory implant manipulator, currently being developed for use in this surgical context

- In the described assessment, the most important shortcoming of this system was a relatively large difference between the planned and the actual drilled trajectories, resulting in contact with the facial nerve

- Insufficient rigidity of the robotic system was a major source of error, to be improved by further development

The most important shortcoming of the current study was the relatively large difference between the planned and the actual drilled trajectories, which resulted in the keyhole drill site contacting the facial nerve. Insufficient rigidity of the robotic system was identified as one major source of error. This will be improved in the future by the use of an additional articulated arm between the base of the robot and the dental bite-block. Other expected improvements include the use of flat panel volume tomography for imaging ${ }^{10}$ and fiducial markers for registration. ${ }^{11}$

\section{Conclusion}

The auditory implant manipulator is a miniaturised robotic instrument manipulator designed to support the surgeon during the insertion of cochlear implants and other implantable hearing devices. We present the clinical work sequence required to generate a mastoid keyhole access site for cochlear implantation. Although additional work is necessary to improve the system, our preliminary results indicate that the auditory implant manipulator has the potential to perform such surgery as a keyhole procedure.

\section{Acknowledgements}

This study was supported by the Commission for Technology and Innovation, Switzerland (grant number KTI 8075), the National Center for Competence in Research Computer Aided and Medical Guided Intervention, Switzerland (grant number NCCR S1NF40111383 ) and the companies Phonak (Stäfa, Switzerland), Cochlear (Sydney, Australia) and Bienair (Biel, Switzerland).

\section{References}

1 Kronenberg J, Migirov L, Dagan T. Suprameatal approach: new surgical approach for cochlear implantation. J Laryngol Otol 2001;115:283-5

2 Hausler R. Cochlear implantation without mastoidectomy: the pericanal electrode insertion technique. Acta Otolaryngol 2002;122:715-19

3 Doshi J, Youngs R. Navigational systems in rhinology: should we all be using them? J Laryngol Otol 2007;121:818-21

4 Hepworth EJ, Bucknor M, Patel A, Vaughan WC. Nationwide survey on the use of image-guided functional endoscopic sinus surgery. Otolaryngol Head Neck Surg 2006;135:68-73

5 Tabaee A, Hsu AK, Shrime MG, Rickert S, Close LG. Quality of life and complications following image-guided endoscopic sinus surgery. Otolaryngol Head Neck Surg 2006;135:76-80

6 Caversaccio M, Langlotz F, Nolte LP, Hausler R. Impact of a self-developed planning and self-constructed navigation system on skull base surgery: 10 years experience. Acta Otolaryngol 2007;127:403-7

7 Brett PN, Taylor RP, Proops D, Coulson C, Reid A, Griffiths MV. A surgical robot for cochleostomy. Conf Proc IEEE Eng Med Biol Soc 2007; 2007:1229-32

8 Coulson CJ, Reid AP, Proops DW, Brett PN. ENT challenges at the small scale. Int J Med Robot 2007;3:91-6

9 Rothbaum DL, Roy J, Stoianovici D, Berkelman P, Hager GD, Taylor RH et al. Robot-assisted stapedotomy: micropick fenestration of the stapes footplate. Otolaryngol Head Neck Surg 2002;127:417-26

10 Majdani O, Bartling SH, Leinung M, Stover T, Lenarz M, Dullin $\mathrm{C}$ et al. A true minimally invasive approach for cochlear implantation: high accuracy in cranial base navigation through flatpanel-based volume computed tomography. Otol Neurotol 2008;29:120-3

11 Labadie RF, Noble JH, Dawant BM, Balachandran R, Majdani O, Fitzpatrick JM. Clinical validation of percutaneous cochlear implant surgery: initial report. Laryngoscope 2008;118:1031-9

12 Maassen MM, Malthan D, Stallkamp J, Schafer A, Dammann F, Schwaderer E et al. Laser-based quality assurance for robotassisted milling at the base of the skull [in German]. HNO 2006;54:105-11

13 Klenzner T, Ngan CC, Knapp FB, Knoop H, Kromeier J, Aschendorff A et al. New strategies for high precision surgery 
of the temporal bone using a robotic approach for cochlear implantation. Eur Arch Otorhinolaryngol 2009;266:955-60

14 Federspil PA, Geisthoff UW, Henrich D, Plinkert PK. Development of the first force-controlled robot for otoneurosurgery. Laryngoscope 2003;113:465-71

15 Zheng G, Caversaccio M, Bachler R, Langlotz F, Nolte LP, Hausler R. Frameless optical computer-aided tracking of a microscope for otorhinology and skull base surgery. Arch Otolaryngol Head Neck Surg 2001;127:1233-8

16 Rudolph T, Puls M, Anderegg C, Ebert L, Broehan M, Rudin A et al. MARVIN: a medical research application framework based on open source software. Comput Methods Programs Biomed 2008;91:165-74

17 Nauer CB, Eichenberger A, Dubach P, Gralla J, Caversaccio M. CT radiation dose for computer-assisted endoscopic sinus surgery: dose survey and determination of dose-reduction limits. AJNR Am J Neuroradiol 2009;30:617-22

18 Muren C, Wadin K, Wilbrand HF. Anatomic variations of the chorda tympani canal. Acta Otolaryngol 1990;110:262-5
19 Noble JH, Warren FM, Labadie RF, Dawant BM. Automatic segmentation of the facial nerve and chorda tympani in CT images using spatially dependent feature values. Med Phys 2008;35:5375-84

Address for correspondence:

Prof Dr Marco Caversaccio,

Chairman,

University Department of ENT,

Head and Neck Surgery, Inselspital/University Bern,

3010 Bern, Switzerland

Fax: + 41316328808

E-mail: marco.caversaccio@insel.ch

Prof Dr M Caversaccio takes responsibility for the integrity of the content of the paper

Competing interests: None declared 